\title{
Electric fields associated with transient surface currents
}

\section{McAllister, lain Wilson}

\section{Published in:}

Journal of Applied Physics

Link to article, DOI:

10.1063/1.350925

Publication date:

1992

\section{Document Version}

Publisher's PDF, also known as Version of record

Link back to DTU Orbit

Citation (APA):

McAllister, I. W. (1992). Electric fields associated with transient surface currents. Journal of Applied Physics, 71(7), 3633-3635. https://doi.org/10.1063/1.350925

\section{General rights}

Copyright and moral rights for the publications made accessible in the public portal are retained by the authors and/or other copyright owners and it is a condition of accessing publications that users recognise and abide by the legal requirements associated with these rights.

- Users may download and print one copy of any publication from the public portal for the purpose of private study or research.

- You may not further distribute the material or use it for any profit-making activity or commercial gain

- You may freely distribute the URL identifying the publication in the public portal

If you believe that this document breaches copyright please contact us providing details, and we will remove access to the work immediately and investigate your claim. 


\title{
Electric fields associated with transient surface currents
}

\author{
I. W. McAllister \\ Physics Laboratory II, Building 309B, The Technical University of Denmark, DK-2800 Lyngby, Denmark
}

\begin{abstract}
(Received 6 February 1991; accepted for publication 19 December 1991)
\end{abstract}

\begin{abstract}
The boundary condition to be fulfilled by the potential functions associated with a transient surface current is derived and expressed in terms of generalized orthogonal coordinates.

From the analysis, it can be deduced that the use of the method of separation of variables is restricted to three surface geometries, viz. a plane, a circular cylinder, and a sphere.
\end{abstract}

In many situations of practical interest, not only do the surfaces of insulating materials exhibit a finite surface conductivity, but they frequently acquire a charge. Owing to surface leakage currents, this charge disperses over the surface through a period of time. Such behavior has recently been examined by Curzon. ${ }^{1-3}$ In these studies, however, the general field theory behind the derivation of the potential solutions is not discussed. In this communication, the basis for analyzing transient surface current field problems is presented, and the separation of variables method is used to derive the boundary condition to be fulfilled at the surface.

Subsequently, the validity of this approach for the present class of boundary value problems is examined, and it is concluded that the use of this method of solution is restricted to three interface geometries, viz. planar, circular cylindrical, and spherical. The occurrence of these particular geometries in the problems examined by Curzon is thus not unexpected (see Refs. 2, 3, and 1, respectively).

In relation to the present analysis we employ generalized orthogonal coordinates $u_{i}(i=1,2,3)$, and we consider the surface in question to be the interface between medium "a" and medium "b." Hence if the interface is taken to be a surface of constant $u_{1}(=\alpha)$, then $u_{2}$ and $u_{3}$ represent surface coordinates upon this interface.

For the situations under discussion, it is convenient to assume that the bulk conductivity $\gamma$ of the insulating materials is much less significant than the surface conductivity $\Gamma$ at the interface. This assumption implies that the conduction field problem reduces to one of a surface current. In such circumstances, the general continuity equation

$$
\operatorname{div} \mathbf{J}+\frac{\partial \rho}{\partial t}=0
$$

(where $\mathbf{J}$ is the volume current density, $\rho$ the volume charge density, and $t$ time) must be replaced by the equivalent surface continuity equation, i.e.,

$$
\operatorname{Div} \mathbf{K}+\frac{\partial \sigma}{\partial t}=0
$$

where $\mathbf{K}$ and $\sigma$ are the surface current density and surface charge density, respectively. This latter continuity relationship is used to develop the boundary condition for the field solution.

The term Div $\mathbf{K}$ represents the surface divergence of $\mathbf{K}$, which is defined as ${ }^{4}$

$$
\operatorname{Div} \mathbf{K}=\lim _{\Delta A \rightarrow 0}\left(\frac{1}{\Delta A} \int_{c} \mathbf{K} \cdot \mathbf{m} d s\right),
$$

where $\mathbf{m}$ is an outward directed unit vector normal to the closed curve $c$ and tangential to the surface in question. The curve $c$ bounds the elemental surface area $\Delta A$, and $s$ is a distance coordinate along $c$. With reference to the orthogonal surface coordinates $u_{2}$ and $u_{3}, \mathbf{K}$ can be expressed as

$$
\mathbf{K}=\mathbf{a}_{2} K_{2}+\mathbf{a}_{3} K_{3},
$$

where $a_{2}$ and $a_{3}$ represent unit vectors, and if we consider a curvilinear rectangle in the surface $u_{1}=$ constant, then Div $\mathbf{K}$ is given by

$$
\begin{aligned}
\operatorname{Div} \mathbf{K}= & \left(g_{22} g_{33}\right)^{-1 / 2}\left[\frac{\partial}{\partial u_{2}}\left(\left(g_{33}\right)^{1 / 2} K_{2}\right)\right. \\
& \left.+\frac{\partial}{\partial u_{3}}\left(\left(g_{22}\right)^{1 / 2} K_{3}\right)\right],
\end{aligned}
$$

where $g_{22}$ and $g_{33}$ are the surface metric coefficients. As the surfaces of interest in the present discussion are associated with generalized orthogonal coordinates, we have

$$
g_{i i}=\left(\frac{\partial x}{\partial u_{i}}\right)^{2}+\left(\frac{\partial y}{\partial u_{i}}\right)^{2}+\left(\frac{\partial z}{\partial u_{i}}\right)^{2}
$$

Following the above assumption concerning the bulk conductivities, the two media will be charge-free and thus the potential distribution in each of them must obey Laplace's equation. Hence we assume that the potential function $\Phi$ can be expressed as

$$
\Phi\left(u_{1}, u_{2}, u_{3}, t\right)=\varphi\left(u_{1}, u_{2}, u_{3}\right) T(t),
$$

with

$$
\varphi\left(u_{1}, u_{2}, u_{3}\right)=U\left(u_{1}\right) V\left(u_{2}\right) W\left(u_{3}\right)
$$

being a solution of Laplace's equation. By expressing $\Phi$ and $\varphi$ in this manner, we have implemented the first step in applying the separation of variables technique. For any coordinate system, the necessary and sufficient conditions to be satisfied with respect to the separability of Laplace's equation are discussed in Moon and Spencer. ${ }^{5}$ To fulfill the field boundary conditions at the interface $u_{1}=\alpha$, we must have $\Phi_{a}\left(\alpha, u_{2}, u_{3}, t\right)=\Phi_{b}\left(\alpha, u_{2}, u_{3}, t\right)$. This condition is obtained if $\Phi_{a}=U_{a} V W T, \Phi_{b}=U_{b} V W T$, and $U_{a}(\alpha)$ $=U_{b}(\alpha)$. Such solutions imply that $\partial \Phi_{a} / \partial u_{2}=\partial \Phi_{b} / \partial u_{2}$ and $\partial \Phi_{a} / \partial u_{3}=\partial \Phi_{b} / \partial u_{3}$ at the interface. 
With respect to the first term of (2), the surface current density $K$ is related to the tangential electric field strength $\mathbf{E}_{t}$ at the surface by

$$
\mathbf{K}=\Gamma \mathbf{E}_{t}
$$

and thus in general we have

$$
\operatorname{Div} \mathbf{K}=\Gamma \operatorname{Div} \mathbf{E}_{t}+\mathbf{E}_{\boldsymbol{t}} \cdot \operatorname{Grad} \Gamma,
$$

where Grad $\Gamma$ represents the surface gradient of $\Gamma$, i.e.,

$$
\operatorname{Grad} \Gamma=\mathbf{a}_{2}\left(g_{22}\right)^{-1 / 2} \frac{\partial \Gamma}{\partial u_{2}}+\mathbf{a}_{3}\left(g_{33}\right)^{-1 / 2} \frac{\partial \Gamma}{\partial u_{3}} \text {. }
$$

The surface conductivity of insulating materials is usually strongly influenced by the local physical and electrical environment of the material, and thus an inhomogeneous $\Gamma$ variation is liable to be the norm rather than the exception. However, in keeping with Curzon, ${ }^{1-3}$ we will assume for the present discussion that $\Gamma$ is constant across the surface, and thus, as $E_{t}$ may be expressed as

$$
\mathbf{E}_{t}=\mathbf{a}_{2} E_{2}+\mathbf{a}_{3} E_{3},
$$

(10) reduces to give

$$
\begin{aligned}
\operatorname{Div} \mathbf{K}= & \Gamma\left(g_{22} g_{33}\right)^{-1 / 2}\left[\frac{\partial}{\partial u_{2}}\left(\left(g_{33}\right)^{1 / 2} E_{2}\right)\right. \\
& \left.+\frac{\partial}{\partial u_{3}}\left(\left(g_{22}\right)^{1 / 2} E_{3}\right)\right] .
\end{aligned}
$$

With reference to generalized coordinates, however, the $E$ components are given by

$$
E_{i}=-\left(g_{i i}\right)^{-1 / 2} \frac{\partial \Phi}{\partial u_{i}},
$$

where $\Phi$ represents the potential in question. Hence by combining (13) and (14) we obtain

$$
\begin{aligned}
\operatorname{Div} \mathbf{K}= & -\Gamma\left(g_{22} g_{33}\right)^{-1 / 2}\left\{\frac{\partial}{\partial u_{2}}\left[\left(\frac{g_{33}}{g_{22}}\right)^{1 / 2} \frac{\partial \Phi}{\partial u_{2}}\right]\right. \\
& \left.+\frac{\partial}{\partial u_{3}}\left[\left(\frac{g_{22}}{g_{33}}\right)^{1 / 2} \frac{\partial \Phi}{\partial u_{3}}\right]\right\} .
\end{aligned}
$$

As $\Phi_{a}\left(\alpha, u_{2}, u_{3}, t\right)=\Phi_{b}\left(\alpha, u_{2}, u_{3}, t\right)$ at the interface, $\mathbf{E}_{t}$ can be derived from either $\Phi_{a}$ or $\Phi_{b}$. We have indicated this situation by omitting the subscripts in (15), and will adopt this procedure subsequently when appropriate.

When expressed in terms of generalized orthogonal coordinates, the scalar Laplacian becomes ${ }^{5}$

$$
\begin{aligned}
\nabla^{2} \Phi= & g^{-1 / 2}\left[\frac{\partial}{\partial u_{1}}\left(\frac{g^{1 / 2}}{g_{11}} \frac{\partial \Phi}{\partial u_{1}}\right)+\frac{\partial}{\partial u_{2}}\left(\frac{g^{1 / 2}}{g_{22}} \frac{\partial \Phi}{\partial u_{2}}\right)\right. \\
& \left.+\frac{\partial}{\partial u_{3}}\left(\frac{g^{1 / 2}}{g_{33}} \frac{\partial \Phi}{\partial u_{3}}\right)\right],
\end{aligned}
$$

where $g=g_{11} g_{22} g_{33}$. An examination of (16) and (15) suggests that by restricting the value of $g_{11}$ to unity, simplification of the expression for Div $\mathbf{K}$ can be achieved. For example, as both $\Phi_{a}$ and $\Phi_{b}$ are solutions of Laplace's equation then with $g_{11}=1$ we obtain from (16) the relationship

$$
\begin{gathered}
\frac{\partial}{\partial u_{2}}\left[\left(\frac{g_{33}}{g_{22}}\right)^{1 / 2} \frac{\partial \Phi}{\partial u_{2}}\right]+\frac{\partial}{\partial u_{3}}\left[\left(\frac{g_{22}}{g_{33}}\right)^{1 / 2} \frac{\partial \Phi}{\partial u_{3}}\right] \\
=-\frac{\partial}{\partial u_{1}}\left(\left(g_{22} g_{33}\right)^{1 / 2} \frac{\partial \Phi}{\partial u_{1}}\right) .
\end{gathered}
$$

Upon substitution of (17) and (7) into (15) and differentiating we obtain finally

$$
\operatorname{Div} \mathbf{K}=\Gamma\left(g_{22} g_{33}\right)^{-1 / 2} \frac{\partial}{\partial u_{1}}\left(\left(g_{222} g_{33}\right)^{1 / 2} \frac{\partial \varphi}{\partial u_{1}}\right) T .
$$

A second boundary condition to be fulfilled at the interface is the continuity of the normal component of the electric flux density $D$, i.e., we have

$$
\sigma=\left(\mathbf{D}_{a}-\mathbf{D}_{b}\right) \cdot \mathbf{n},
$$

where $\mathbf{n}$ is a unit vector normal to the interface in the direction " $b$ " to " $a$." This orientation is also associated with increasing values of $u_{1}$, so that $u_{1 a} \geqslant u_{1 b}$. In the present analysis, the normal component of $\mathbf{D}$ is given by $\epsilon E_{1}$, where $\epsilon$ is the permittivity, and hence at the interface we have

$$
\sigma=-\left(g_{11}\right)^{-1 / 2}\left(\epsilon_{a} \frac{\partial \Phi_{a}}{\partial u_{1}}-\epsilon_{b} \frac{\partial \Phi_{b}}{\partial u_{1}}\right)
$$

or, with reference to (7) and (2),

$$
\frac{\partial \sigma}{\partial t}=\left(g_{11}\right)^{-1 / 2}\left(\epsilon_{b} \frac{\partial \varphi_{b}}{\partial u_{1}}-\epsilon_{a} \frac{\partial \varphi_{a}}{\partial u_{1}}\right) \frac{d T}{d t} .
$$

Consequently the surface continuity equation for the interface can be expressed in terms of generalized coordinates as

$$
\begin{gathered}
\Gamma\left(g_{22} g_{33}\right)^{-1 / 2} \frac{\partial}{\partial u_{1}}\left(\left(g_{22} g_{33}\right)^{1 / 2} \frac{\partial \varphi}{\partial u_{1}}\right) T \\
+\left(\epsilon_{b} \frac{\partial \varphi_{b}}{\partial u_{1}}-\epsilon_{a} \frac{\partial \varphi_{a}}{\partial u_{1}}\right) \frac{d T}{d t}=0 .
\end{gathered}
$$

For $g_{11}=1$, this expression represents the general boundary condition to be fulfilled by $\Phi_{a}$ and $\Phi_{b}$ at the interface $\left(u_{1}=\alpha\right)$.

As each of the bracketed terms is independent of $t$, it is clear that (22) can be written as

$$
\frac{d T}{d t}+\lambda T=0
$$

with

$$
\lambda=\frac{\Gamma\left(g_{22} g_{33}\right)^{-1 / 2}\left(\partial / \partial u_{1}\right)\left[\left(g_{22} g_{33}\right)^{1 / 2}\left(\partial \varphi / \partial u_{1}\right)\right]}{\epsilon_{b}\left(\partial \varphi_{b} / \partial u_{1}\right)-\epsilon_{a}\left(\partial \varphi_{a} / \partial u_{1}\right)} .
$$

The general solution for (23) is of course

$$
T=B \exp (-\lambda t),
$$

where $B$ is a constant.

In deriving (25), the basic assumption is that the separation of variables method can be employed to obtain solutions for the potential functions $\Phi_{a}$ and $\Phi_{b}$. Hence as $T$ is a function of $t$ alone, this assumption implies that, with respect to the interface, $\lambda$ must have a constant valuc. 
For Laplace's equation to be simply separable, i.e., to be expressed as in ( 8 ), the necessary and sufficient conditions are ${ }^{5}$

$$
\frac{g_{i i}}{g_{j j}}=\frac{M_{j 1}}{M_{i 1}} \quad \text { for } j=1,2,3 \text { and } j \neq i \text {, }
$$

and

$$
\frac{g^{1 / 2}}{g_{i i}}=f_{1} f_{2} f_{3} M_{i 1}
$$

where $M_{i 1}\left(u_{j}, u_{k}\right)$ and $M_{j 1}\left(u_{i}, u_{k}\right)$ are the cofactors of the $i$ th and $j$ th elements in the first column of the Stäckel determinant $(k=1,2,3$ and $k \neq j, k \neq i)$. The determinant arises from the technique of separating a three-dimensional partial differential equation into three ordinary differential equations, see Moon and Spencer ${ }^{5}$ for details. The principal characteristic of this determinant is that each row contains functions of only one variable (or constants). $f_{1}\left(u_{1}\right)$, $f_{2}\left(u_{2}\right)$ and $f_{3}\left(u_{3}\right)$ are functions which are introduced to fulfill the requirement of separability and are deduced from the coordinate system in question via the metric coefficients and the separability conditions. Full information about the various functions for all the relevant coordinate systems is to be found in the Field Theory Handbook. ${ }^{6}$

Thus upon substituting (8) and employing (27), wc can express (24) as

$$
\lambda=\frac{\left(\Gamma / f_{1}\right)\left(\partial / \partial u_{1}\right)\left[f_{1}\left(\partial U / \partial u_{1}\right)\right]}{\epsilon_{b}\left(\partial U_{b} / \partial u_{1}\right)-\epsilon_{a}\left(\partial U_{a} / \partial u_{1}\right)} .
$$

Both $U$ and $f_{1}$ are, however, functions of $u_{1}$ alone and hence at the interface $\left(u_{1}=\alpha\right)$ these parameters will have constant values. Consequently $\lambda$ is a constant.

A study of the Field Theory Handbook ${ }^{6}$ indicates that from the 19 distinct surfaces generated by the 11 coordinate systems which are simply separable, only three surfaces fulfill the stated $g_{11}$ requirement; viz. a plane, a circular cylinder, and a sphere. For each of these geometries, it is found that, with the relevant coordinate system (see Table I), $g_{11}=1$. Although the three geometries are also generated by other coordinate systems, ${ }^{6}$ we find in each case that Laplace's equation is not simply separable and that $g_{11} \neq 1$.

An expression for $\lambda$ can be derived without assuming $g_{11}=1$. From such an expression, however, it is possible to show that, in general, $\lambda$ would be a function of the surface coordinates $u_{2}$ and $u_{3}$. In such circumstances, the separation of variables method ceases to be valid. Consequently, the use of this method to derive solutions for $\Phi_{a}$ and $\Phi_{b}$ is
TABLE I. Coordinate systems associated with simple separability and $g_{11}=1 . \mu$ is the coordinate representing the circular cylindrical surface. Similarly $r$ represents the spherical surface.

\begin{tabular}{llc}
\hline \hline Surface geometry & Coordinate system & $f_{1}$ \\
\hline plane & rectangular & 1 \\
& circular-cylinder & 1 \\
& elliptic-cylinder & 1 \\
& parabolic-cylinder & 1 \\
circular cylinder & circular-cylinder & $r$ \\
sphere & spherical & $r^{2}$ \\
& conical & $r^{2}$ \\
\hline \hline
\end{tabular}

restricted to the three surface geometries involving the coordinate systems listed in Table I.

The constant $\lambda$ associated with the decay of the field produced by a transient surface current is given by (28). However, in deriving a potential solution for a specific set of boundary conditions, it is frequently necessary to employ a superposition of general solutions to fulfill these boundary conditions. Depending upon the situation, the particular potential solution may be expressed as either an infinite series, or an infinite integral. In such cases, (28) cannot be used directly to determine $\lambda$. Rather reference must be made to (22) which, on introducing the separability conditions, reduces to give

$$
\frac{\Gamma}{f_{1}} \frac{\partial}{\partial u_{1}}\left(f_{1} \frac{\partial \varphi}{\partial u_{1}}\right) T+\left(\epsilon_{b} \frac{\partial \varphi_{b}}{\partial u_{1}}-\epsilon_{a} \frac{\partial \varphi_{a}}{\partial u_{1}}\right) \frac{d T}{d t}=0
$$

as the interface $\left(u_{1}=\alpha\right)$ boundary condition. The relevant $f_{1}$ functions for the three surfaces of interest are listed in Table I.

It may be concluded that the studies of Curzon ${ }^{1-3}$ cannot be extended to other surface geometries using the separation of variables method. In the present analysis, the restrictions encountered with this method when dealing with transient surface currents are brought clearly into focus.

The author wishes to thank P. W. Karlsson for several illuminating discussions on the concept of surface divergence.

${ }^{1}$ F. L. Curzon, J. Appl. Phys. 65, 4500 (1989).

${ }^{2}$ F. L. Curzon, J. Phys. D 22, 891 (1989).

${ }^{3}$ F. L. Curzon, J. Appl. Phys. 66, 1517 (1989).

${ }^{4}$ E. Martensen, Potentialtheorie (Teubncr, Stuttgart, 1968).

${ }^{5}$ P. Moon and D. E. Spencer, Field Theory for Engineers (Van Nostrand, Princeton, 1961), Chap. 11.

${ }^{6}$ P. Moon and D. E. Spencer, Fleld Theory Handbook (Springer, Berlin, 1961). 